\title{
Optimising the carbon 13 sucrose breath test for the assessment of environmental enteric dysfunction
}

\author{
R. J. Schillinger ${ }^{1}$, D. J. Morrison ${ }^{2}$ and C. A. Edwards ${ }^{1}$ \\ ${ }^{1}$ College of Medical, Veterinary and Life Sciences, University of Glasgow, Glasgow, Scotland, G12 8QQ and \\ ${ }^{2}$ Scottish Universities Environmental Research Centre, University of Glasgow, East Kilbride, Scotland, G75 OQF
}

Environmental enteric dysfunction (EED) is a complex disorder characterised by structural and functional aberrations within the small intestine ${ }^{(1)}$. It is hypothesised that EED is the result of repeated enteric infections combined with under-nutrition and is prevalent throughout low and middle income countries. EED is thought to compromise linear growth potentially leading to stunting in infants ${ }^{(2)}$. Stunting currently affects 144 million children globally under the age of $5^{(3)}$. ${ }^{13} \mathrm{C}$-sucrose from naturally enriched maize sources has previously been used in a breath test to assess intestinal sucrase activity as a marker of mucosal integrity and function in enteric enteropathy ${ }^{(4)}$. However, this test requires up to $20 \mathrm{~g}$ of naturally ${ }^{13} \mathrm{C}$ enriched sucrose to achieve acceptable signal-to-noise in breath ${ }^{13} \mathrm{CO}_{2}$, making the test unsuitable for routine use in infants. The current study aimed to develop a new ${ }^{13} \mathrm{C}$ sucrose breath test (SBT) protocol using commercially available, highly enriched ${ }^{13} \mathrm{C}$ Sucrose to facilitate a much smaller test dose.

This was a randomised crossover trial of 19 healthy adults recruited in Glasgow, UK. All participants completed a baseline SBT with $20 \mathrm{~g}$ naturally enriched sucrose before being randomly assigned to two groups; receiving $20 \mathrm{~g}$ unlabelled (beet) sucrose $+50 \mathrm{mg}$ tracer $(n=8)$ or $50 \mathrm{mg}$ tracer dose alone $(n=11)$. Participants remained allocated to their group and were given in a random repeated order ${ }^{13} \mathrm{C}_{12}$-Sucrose, ${ }^{13} \mathrm{C}_{6}$ Sucrose $\left({ }^{13} \mathrm{C}_{6}\right.$-Fructose) and ${ }^{13} \mathrm{C}_{6}$ Sucrose $\left({ }^{13} \mathrm{C}_{6}\right.$-Glucose) to assess the effect of labelling position on breath recovery parameters with at least 3 days washout between tests.

Results were expressed as Area Under the Curve (AUC), time of maximum breath ${ }^{13} \mathrm{CO}_{2}$ enrichment ( $\mathrm{T}_{\max }$, hrs), cumulative percentage dose recovered at 90 mins (cPDR90, \%) and time at which 50\% AUC had been expired in breath $13 \mathrm{CO} 2$ ( $\mathrm{T}_{1 / 2}$, hrs).

Highly enriched ${ }^{13} \mathrm{C}_{12}$ sucrose yielded significantly improved ${ }^{13} \mathrm{C}$ breath signal to noise compared to the naturally enriched sucrose dose (x20). There were no significant differences in AUC, cPDR90, $\mathrm{T}_{\max }$ or $\mathrm{T}_{1 / 2}$ between ${ }^{13} \mathrm{C}_{12}$ sucrose and naturally enriched sucrose (all $\mathrm{P}>0.05$ ). Altering the position of the ${ }^{13} \mathrm{C}$ label changed kinetics of breath ${ }^{13} \mathrm{C}$ excretion. ${ }^{13} \mathrm{CO}_{2}$ appeared more rapidly with ${ }^{13} \mathrm{C}_{6}$ sucrose $\left({ }^{13} \mathrm{C}_{6}\right.$-fructose) compared with ${ }^{13} \mathrm{C}_{6}$ sucrose $\left({ }^{13} \mathrm{C}_{6}\right.$-glucose $)\left(\mathrm{T}_{\max },(\mathrm{SD})=1.29(0.44)\right.$ vs. $1.87(0.22), \mathrm{P}<0.05$ and cPDR $90(\mathrm{SD})$ $=15.95$ (3.88) vs. 12.38 (2.31), $\mathrm{P}<0.05$ respectively). Addition of $20 \mathrm{~g}$ unlabelled sucrose appeared to slow down ${ }^{13} \mathrm{C}$ sucrose digestion with significantly lower cPDR90 for all highly enriched variants (all $\mathrm{P}<0.05)$.

A dose of $50 \mathrm{mg}{ }^{13} \mathrm{C}_{12}$-sucrose gave excellent signal-to-noise in breath ${ }^{13} \mathrm{CO}_{2}$ and there was no statistically significant difference in breath parameters compared with the naturally enriched SBT. The ${ }^{13} \mathrm{C}$ labelling pattern impacted the kinetics of ${ }^{13} \mathrm{C}$ recovery in breath probably reflecting intermediary metabolism. A minimal dose of ${ }^{13} \mathrm{C}_{12}$-sucrose is likely to be suitable for studies in infants and children.

1. Crane RJ, Jones KD \& Berkley JA (2015) Food Nutr Bull 36, S76-S87.

2. Butler RN, Kosek M, Krebs NF et al. (2017). J Pediatr Gastroenterol Nutr 64, 1, 8-14

3. World Health Organisation (2020). Joint child malnutrition estimates.

4. Ritchie BK, Brewster DR, Davidson GP et al. (2009). Pediatrics 124, 2, 620-626 